\title{
Tracheal intubation with channeled vs. non-channeled videolaryngoscope blades
}

\author{
Peter Biro ${ }^{1}$, Martin Schlaepfer ${ }^{2}$
}

${ }^{1}$ Institute of Anaesthesiology, University Hospital Zurich, Switzerland

${ }^{2}$ Institute of Anaesthesiology, Institute of Physiology, University and University Hospital Zurich, Switzerland

\begin{abstract}
Study objective. Videolaryngoscopes can be fitted either with channeled or non-channeled blades, which may result in a different performance and success of tracheal intubation. We investigated the

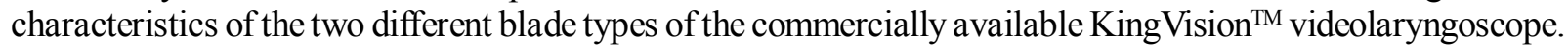

Design. A prospective, randomized, single center investigation study in a urological operation unit of a tertiary hospital.

Subjects and Methods. Forty adult patients undergoing elective urological surgery in general anaesthesia with tracheal intubation were randomly allocated into group 1 (channeled videolaryngoscopy, $n=20$ ) and group 2 (non-channeled videolaryngoscopy, $\mathrm{n}=20$ ). We measured the times from laryngoscope insertion to recognize the glottis and to conclude tracheal intubation. The number of laryngoscopy/intubation attempts and the degree of visual glottis exposure on a visual analog scale from 0 (glottis not visible) to 10 (glottis fully visible) was assessed. The lowest $\mathrm{SpO}_{2}$ value during airway management was recorded.

Results. There was no statistically significant difference in biometric data between the 2 groups. The time from the laryngoscope insertion to glottis recognition with the non-channeled blades was $5(4-8) \mathrm{s}$ as compared to the channeled ones with $11(7-14) \mathrm{s}$ (median and range; $p=0.01)$. Intubation duration was shorter with the channeled blades 17 (12-27) s vs. 29 (25-51) s (median and range; $p<0.001)$. Number of laryngoscopy/intubation attempts, grades for glottis visibility, intubation difficulty were not different. The lowest $\mathrm{SpO}_{2}$ was $98 \%$ in both groups.

Conclusions. Videolaryngoscopic glottis recognition time was longer and the total time to secure the airway was shorter with the channeled blades.
\end{abstract}

Keywords: Intubation; videolaryngoscopy; laryngoscope blades; channeled, non-channeled

Rom J Anaesth Intensive Care 2018; 25: 97-101

\section{Introduction}

Videolaryngoscopy has gained a strong foothold in routine anaesthesia practice and became a recommended alternative technique in cases of expected difficult airway situations [1-4]. Meanwhile, the large number of available videolaryngoscopes (VL) and related equipment exceeds the ability of normal professionals to test them and to identify the optimal device for their specific demands. The basic blade type, which may be either non-channeled or channeled, represents major practical and methodological differences between VL. This distinction is of clinical relevance, since it requires different handling and may affect the success or failure of its use.

Earlier versions of VL were designed in a nonchanneled blade configuration. When using these blades, the VL must be held in the left hand and the tracheal tube (TT) has to be steered independently with the right hand. This approach has the advantage for the user that he can freely control the movements and the trajectory of the TT, a circumstance that might 
be preferable for experienced users. The main disadvantage of the non-channeled blade is the more difficult simultaneous handling of the TT and the VL while maintaining the best glottis view on the screen. In addition, during the first phase of TT insertion there is a "blind" period where the TT's tip does not yet appear on the screen. This circumstance may lead to exploratory moves of the TT, which may cost time and cause injuries. Additionally, in order to give the TT the necessary curved shape and stability, a malleable stylet has to be inserted and bended according the shape of the blade's curvature.

Conversely, to facilitate the approach of the TT tip towards the glottis, so-called "channeled" blades have been developed. These are equipped on their right edge with a longitudinal trough (channel), into which the TT is inserted so that its tip becomes permanently visible on the screen. Thus, the TT strictly follows the VL blade. As soon as the glottis opening is in the center of the video image, the TT is advanced forward and should enter the airway without the necessity of being separately steered. After placing the TT into its final position, the user removes the VL by detaching it from the TT. This configuration should enable successful intubation in the hand of less experienced users. The disadvantage of the channeled blade is a bulkier design and the necessity for a larger mouth opening.

In this prospective, randomized, single center investigation, we aimed to investigate time to larynx recognition and time to successful intubation of the non-channeled vs. channeled versions of the commercially available KingVision ${ }^{\mathrm{TM}}$ VL (Ambu A/S, Baltorpbakken 13, 2750 Ballerup, Denmark).

\section{Methods}

In this current work, we aim to investigate the impact of laryngoscopy with channeled vs. nonchanneled blades for tracheal intubation. We analyzed a dataset which was created for a recently accepted article [5] and extracted additional data for this investigation. The local Ethics Committee approved the original study (No. 2016-01657, Chair: Prof. Dr. Peter Meier-Abt, issued on 30 March 2017) and issued an amendment on 23 January 2018 permitting the use of these data for publication. The original trial focused on investigating a novel transillumination technique as an aid for tracheal intubation.

Forty participants were recruited and informed consent on the day before the intervention was obtained. Participants were scheduled for elective urological surgery under general anaesthesia. The patients were assigned to 2 even groups of 20 patients each. The first group was to be intubated using a nonchanneled VL blade, the second group was intubated with a channeled VL blade $[2,6]$. Both disposable blades were designed for the KingVision ${ }^{\mathrm{TM}}$ VL (Figure 1). When using the non-channeled blades, the TT was armed with a malleable guidewire (Shiley ${ }^{\mathrm{TM}}$, Covi$\operatorname{dien}^{\mathrm{TM}}, 15$ Hampshire Street, Ireland) and shaped to a curved form with the same radius as the VL blade [7].

In order to avoid a bias due to differences in professional experience and skill, the same investigator (PB) performed all laryngoscopies and intubations. This "single experienced investigator" study model with one user, who is familiar with the videolaryngoscopic intubation technique in general and the two blade variants in particular, was adopted in order to obtain uninfluenced baseline data regarding the difference between the two blade types.

Induction of anaesthesia, videolaryngoscopy and tracheal intubation were performed in a standardized manner: for anaesthesia induction propofol $2 \mathrm{mg} / \mathrm{kg}$, rocuronium $1 \mathrm{mg} / \mathrm{kg}$ and fentanyl $3 \mu \mathrm{g} / \mathrm{kg}$ were administered. Videolaryngoscopy was initiated after relaxometric confirmation of a sufficient neuro-muscular blockade at a train of four (TOF) count $<1$.

We measured and recorded the characteristics of laryngoscopy and intubation:

- Time from oral insertion of the VL until clear recognition of the glottis opening on the VL screen.

- Number of VL insertion attempts until the best glottis view was achieved.

- Visual exposure of the glottis on the VL screen according a visual analog scale (VAS) ranging from 0 (glottis not visible) to 10 (glottis fully visible).

- Number of tracheal intubation insertion attempts until success (or abortion in the case of 3 attempts).

- Time from oral insertion of the VL to inflating the cuff of the successfully placed TT.

- Subjective degree of difficulty for the entire intubation procedure according a VAS ranging from 0 (very easy) to 10 (very difficult).

- Lowest peripheral oxygen saturation $\left(\mathrm{SpO}_{2}\right)$ during the intubation process.

During the recovery in the post anaesthesia care unit (PACU), all patients were asked whether they had complaints related to the airway manipulations such as dental damage or sore throat. When a sore throat was reported, a second visit and reassessment was scheduled for the next day.

Statistical analyses were performed using GraphPad Prism 7.0d (GraphPad inc. La Jolla, CA). Normality was tested by D'Agostino and Pearson's normality test. As almost all of the dates appear to be skewed, data are presented as median (interquartile range $=$ IQR). Group differences of categorical data (gender 

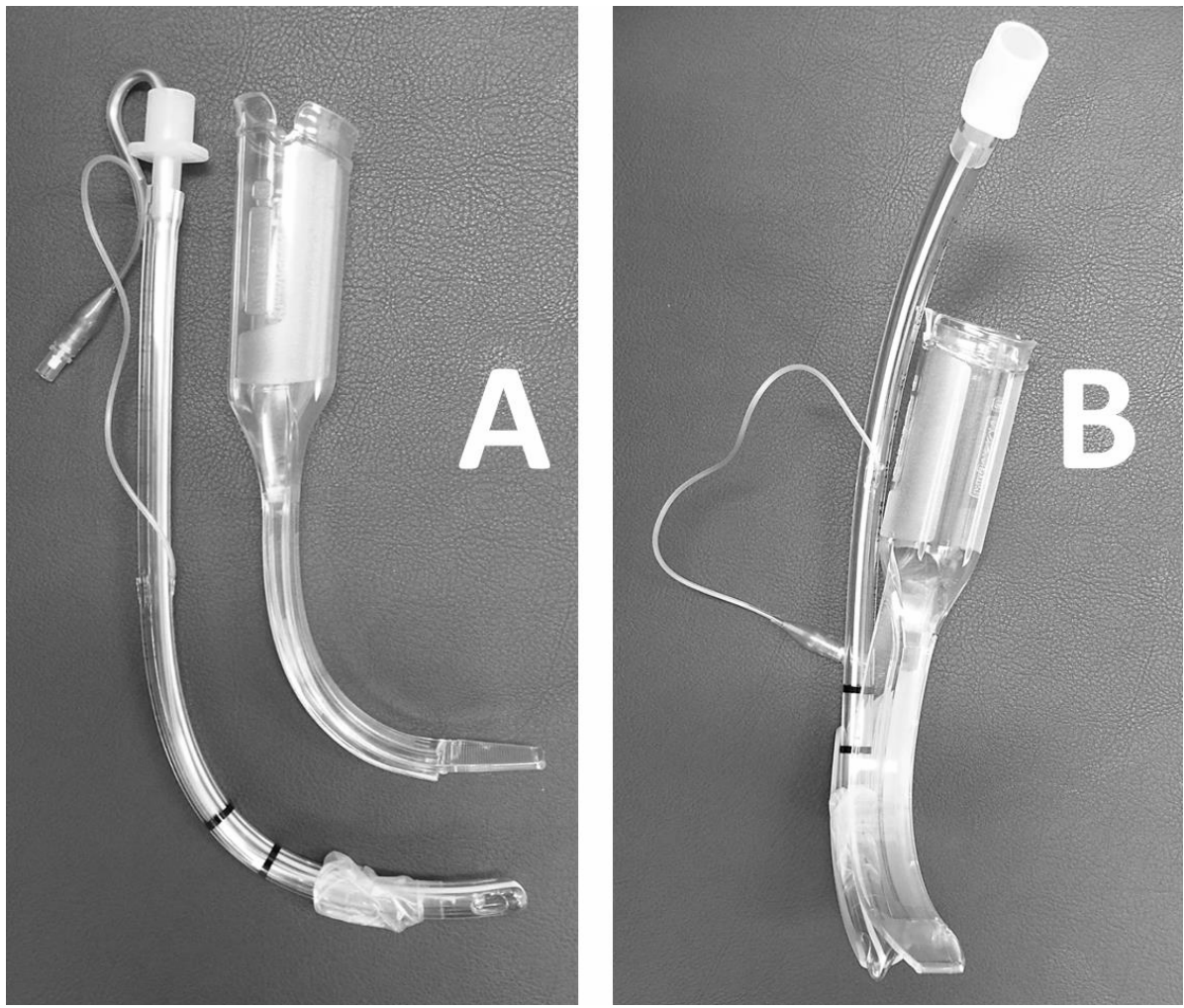

Fig. 1. A: Non-channeled KingVision ${ }^{\mathrm{TM}}$ blade and a suitably curved tracheal tube with a malleable stylet inside; B: Channeled KingVision ${ }^{\mathrm{TM}}$ blade with a tracheal tube inserted in its longitudinal trough. (Image is own work)

distribution, Mallampati grade, ASAclassification) were assessed by the Chi-Square test; all other data were assessed by a two-tailed Mann-Whitney test. Exact $\mathrm{p}$-values were computed and $\mathrm{p}<0.05$ was considered statistically significant.

\section{Results}

Patient characteristics: Patient baseline characteristics with respect to weight, age, gender distribution, neck perimeter and BMI were similar in the two groups (Table 1). There was also no statistically significant difference in the distribution of the patients' Mallampati grades and ASA class distribution.

Larynx recognition: The time to larynx recognition was significantly shorter when using the non-channeled blades as compared to the channeled ones: $5(4-8) \mathrm{s}$ with non-channeled vs. 11 (7-14) s (median and range; $p=0.01)$. The grade of glottis visibility and the number of VL insertions were similar (Table 2).

Intubation performance: The time to successful intubation was significantly longer when using the nonchanneled compared to the channeled blades: 40 (1227) s compared to 20 (25-51) s (median and range; $p$ $<0.001)$. The grade of intubation difficulty and the number of intubation attempts were similar in the two groups (Table 3 ). The lowest peripheral oxygen saturation was similar in both groups: non-channeled 98\% (97-99) vs. channeled 98\% (97-99), (median and range; n.s.).

All intubations could be concluded successfully; a deviation from the original approach was not necessary

Table 1. Biometric characteristics of the investigated patients in the 2 study groups

\begin{tabular}{lccl}
\hline & $\begin{array}{c}\text { Group 1 } \\
\text { Non-channeled }\end{array}$ & $\begin{array}{c}\text { Group 2 } \\
\text { Channeled }\end{array}$ & p \\
\hline Weight $(\mathrm{kg})$ & $82(76-94)$ & $79(75-93)$ & n.s. \\
\hline Age $($ years) & $66(54-71)$ & $66(61-74)$ & n.s. \\
Gender distribution $(\mathrm{m} / \mathrm{f})$ & $15 / 5$ & $19 / 1$ & n.s. \\
Mallampati grade distribution $(\mathrm{n}$ for $1 / 2 / 3 / 4)$ & $7 / 8 / 3 / 2$ & $11 / 9 / 0 / 0$ & n.s. \\
ASA class distribution $(\mathrm{n}$ for $1 / 2 / 3 / 4)$ & $6 / 11 / 3 / 0$ & $2 / 14 / 4 / 0$ & n.s. \\
Neck perimeter $(\mathrm{cm})$ & $41(37-46)$ & $41(38-45)$ & n.s. \\
Body mass index $\left(\mathrm{kg} / \mathrm{m}^{2}\right)$ & $26(24-27)$ & $26(23-29)$ & n.s. \\
\hline
\end{tabular}

ASA $=$ American Society of Anesthesiologists; median (range) or n; n.s. = not significant 
Table 2. Performance of a laryngoscopy in the 2 study groups

\begin{tabular}{|lccc|}
\hline & $\begin{array}{c}\text { Group 1 } \\
\text { Non-channeled }\end{array}$ & $\begin{array}{c}\text { Group 2 } \\
\text { Channeled }\end{array}$ & $p$ \\
\hline Time to glottis recognition (s) & $5(4-8)$ & $11(7-14)$ & 0.01 \\
Grade of glottis visibility (VAS) & $5(4-7)$ & $6(4-7)$ & n.s. \\
Number of VL insertions (n for 1/2/3) & $14 / 5 / 1$ & $15 / 5 / 0$ & n.s. \\
\hline
\end{tabular}

$\mathrm{VAS}=$ visual analog scale; $\mathrm{VL}=$ videolaryngoscope; median (range) or $\mathrm{n}$; n.s. = not significant

Table 3. Intubation performance in the 2 study groups

\begin{tabular}{lccc}
\hline & $\begin{array}{c}\text { Group 1 } \\
\text { Non-channeled }\end{array}$ & $\begin{array}{c}\text { Group 2 } \\
\text { Channeled }\end{array}$ & p \\
\hline Time to successful intubation (s) & $29(25-51)$ & $17(12-27)$ & $<0.001$ \\
\hline Grade of intubation difficulty (VAS ranging from $0=$ easy to $10=$ difficult) & $5(2-7)$ & $5(3-6)$ & n.s. \\
\hline Number of intubation attempts (n for $1 / 2 / 3)$ & $15 / 2 / 3$ & $16 / 3 / 1$ & n.s. \\
\hline
\end{tabular}

VAS $=$ visual analog scale; median (range) or $n$; n.s. $=$ not significant

and no switching from one blade type to the other occurred. There were no complications connected to the management of the airway. At the post-anaesthesia visit, 2 hours after extubation, 3 patients in Group 1 (non-channeled) and 4 patients in Group 2 (channeled) complained about transient sore throat, which disappeared during the first postoperative day. This circumstance did not yield statistically significant differences between the 2 study groups. There was no difference in the subjective assessment of visual exposure of the glottis on the VL screen and the number of laryngoscope insertions (Table 2).

Tracheal intubations showed no statistical differences concerning the number of attempts and the subjective level of difficulty as well as the lowest peripheral saturation during management of the airway. However, the duration to successful conclusion of the entire intubation process (from insertion of the VL until blocking the TT cuff) was significantly shorter with the channeled blades (Table 3).

\section{Discussion}

There is still no conclusive and generally recommended indication regarding which VL blade type is preferable. Alvis et al. compared airway management performance between the non-channeled $\mathrm{McGrath}^{\mathrm{TM}}$ and the channeled KingVision ${ }^{\mathrm{TM}}$ videolaryngoscopes [8]. They found significantly shorter median intubation times ( 17 vs. 38 seconds; $p<0.001$ ), a higher first attempt success rate and less oxygen desaturations $<90 \%$ using the non-channeled blades. However, they compared different VL brands, so that the identified differences could also be a result of different basic designs. In a large prospective multicenter trial on 720 patients, 5 VL types were evaluated by 12 different anaesthe- siologists. Three of the tested instruments (Airtraq ${ }^{\mathrm{TM}}$, Advance $^{\mathrm{TM}}$ and KingVision ${ }^{\mathrm{TM}}$ ) had blades of both basic types and their conclusion was that both blade types showed similar success rates. They also stated that overall performance depended more on the basic design of the involved instruments, rather than on the presence of a channel for tube advancement [9]. These findings are difficult to be interpreted because of the multitude of involved users with various levels of experience, dexterity and different familiarity with the tested devices.

In contrast to these previous studies, the current investigation is based on a "single best user" approach, by which the individual experience and skill does not affect the results. Our study indeed revealed clear differences between the 2 blade types: the time duration to recognize the glottis on the video screen of the $\mathrm{VL}$ was significantly shorter with the non-channeled blade, although the clinical relevance of the $6 \mathrm{~s}$ in average is questionable. The reason for the longer glottis recognition time with the channeled blade is its bulkiness. Otherwise, the laryngoscopy procedure including the glottis exposure was similar in the two groups. In contrast to the prolonged glottis recognition time with channeled blades, we found the time to successful intubation to be faster and the handling easier. This may well be relevant for the ultimate goal of tracheal intubation, thus representing an argument in favor of the channeled blade type. The reason for the faster intubation time (despite the slower larynx recognition time) lies in the declared purpose of the device: the channeled blade makes the more demanding steering of the TT superfluous and omits the "blind" phase during advancement of the TT; these maneuvers are time consuming and therefore the disadvantage in larynx recognition is outweighed by the easiness and promptitude of intubation. 
Recently, a similar study by Krieger et al. compared non-channeled vs. channeled KingVision ${ }^{\mathrm{TM}}$ VL blades [10]. In contrast to our results, they reported that the channeled blades did not make intubation easier and they found even shorter intubation times (median $40 \mathrm{~s}$; inter-quartal range IQR (24-58)) with the nonchanneled blades $(59 \mathrm{~s}(40-74) ; \mathrm{p}=0.03)$. With respect to the glottis visualization, their results are in line with our study, as they also did not find a difference between the 2 groups [10]. Now we have no lucid explanation for the fact that our results are partially opposed to Krieger's report, except the circumstance, that we applied a single user and "best expert" approach, while they involved multiple users.

In the context of these comparative studies, our current one contributes to the discussion regarding the advantages and disadvantages of the two different blades types for videolaryngoscopic intubation. Our study has several strengths and limitations: the main strength is the single user approach, which reduces inter-individual skill differences among multiple users and allows detecting differences in a smaller sample size. However, at the same time this is a certain limitation, since a single user reflects only an individual skill level, which therefore might not be representative for a larger population. A further disadvantage of our study is that we did not include various VL brands, so that our results stand for the impact of this specific instrument only. A multiple user approach with clearly stratified user experience levels and in a bigger patient population would be desirable to reliably appraise the impact of most available VL blade types.

\section{Conclusions}

Our current study suggests that the time to videolaryngoscopic glottis recognition is longer when using a channeled blade, but time to intubation and the total time to secure the airway is shorter. The intended benefit of channeled blades could be confirmed.

Key messages:

- Videolaryngoscope blades are either channeled or non-channeled

- Their design needs different methodological approaches

- Non-channeled blades are easier to insert than channeled ones

- Channeled blades facilitate intubation more than the non-channeled ones

- Channeled blades are more suitable for less experienced users

- Experienced users might prefer the larger flexibility when using non-channeled blades

\section{Conflict of interest}

PB received travel allowances by Merck Sharp \& Dohme and by Acutronic Medical Systems. MS received travel support from Baxter, Switzerland.

\section{Acknowledgement}

This study was unconditionally supported by Guide in Medical (Nazareth, Israel) by providing the KingVisionTM videolaryngoscope and the blades used for this study.

\section{References}

1. Apfelbaum JL, Hagberg CA, Caplan RA, Blitt CD, Connis RT, Nickinovich DG, et al. Practice guidelines for management of the difficult airway: an updated report by the American Society of Anesthesiologists Task Force on Management of the Difficult Airway. Anesthesiology 2013; 118: 251-270. doi: 10.1097/ ALN.0b013e31827773b2

2. Ducharme S, Kramer B, Gelbart D, Colleran C, Risavi B, Carlson $\mathrm{JN}$. A pilot, prospective, randomized trial of video versus direct laryngoscopy for paramedic endotracheal intubation. Resuscitation 2017; 114: 121-126. doi: 10.1016/j.resuscitation. 2017.03.022

3. Lewis SR, Butler AR, Parker J, Cook TM, Smith AF. Videolaryngoscopy versus direct laryngoscopy for adult patients requiring tracheal intubation. Cochrane Database Syst Rev 2016; 11 : CD011136. doi: 10.1002/14651858.CD011136.pub2

4. Parasa M, Yallapragada SV, Vemuri NN, Shaik MS. Comparison of GlideScope video laryngoscope with Macintosh laryngoscope in adult patients undergoing elective surgical procedures. Anesth Essays Res 2016; 10: 245-249. doi: 10.4103/0259-1162.167840

5. Biro P, Fried E, Schlaepfer M, Kristensen MS. A new retrograde transillumination technique for videolaryngoscopic tracheal intubation. Anaesthesia 2018; 73: 474-479. doi: 10.1111/ anae. 14217

6. Valencia JA, Pimienta K, Cohen D, Benitez D, Romero D, Amaya O, et al. A comparison of King Vision video laryngoscopy and direct laryngoscopy as performed by residents: a randomized controlled trial. J Clin Anesth 2016; 35: 571-575. doi: 10.1016/ j.jclinane.2016.07.039

7. Cooper RM. Strengths and limitations of airway techniques. Anesthesiol Clin 2015; 33: 241-255. doi: 10.1016/ j.anclin.2015.02.006

8. Alvis BD, Hester D, Watson D, Higgins M, St Jacques P. Randomized controlled trial comparing the McGrath MAC video laryngoscope with the King Vision video laryngoscope in adult patients. Minerva Anestesiol 2016; 82: 30-35

9. Kleine-Brueggeney M, Greif R, Schoettker P, Savoldelli GL, Nabecker S, Theiler LG. Evaluation of six videolaryngoscopes in 720 patients with a simulated difficult airway: a multicentre randomized controlled trial. Br J Anaesth 2016; 116: 670-679. doi: $10.1093 / \mathrm{bja} / \mathrm{aew} 058$

10. Kriege M, Alflen C, Noppens RR. Using King Vision video laryngoscope with a channeled blade prolongs time for tracheal intubation in different training levels, compared to nonchanneled blade. PLoS One 2017; 12: e0183382. doi: 10.1371/ journal.pone. 0183382 